Document downloaded from:

http://hdl.handle.net/10251/47912

This paper must be cited as:

Comini, M.; Titolo, L.; Villanueva García, A. (2014). Abstract Diagnosis for tccp using a Linear Temporal Logic. Theory and Practice of Logic Programming. 14(4-5):787-801. doi:10.1017/S1471068414000349.

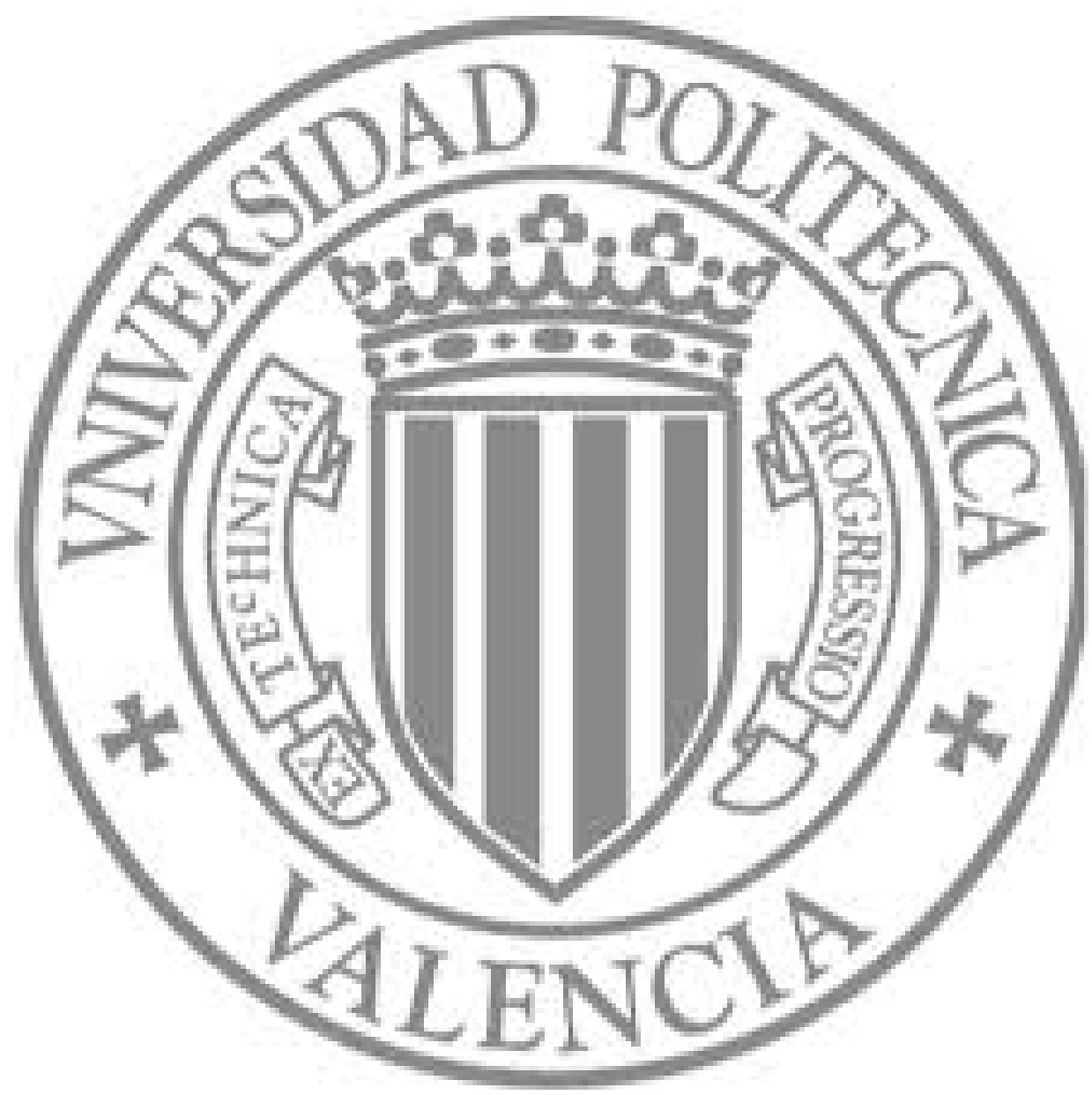

The final publication is available at

http://dx.doi.org/10.1017/S1471068414000349

Copyright Cambridge University Press 


\title{
Abstract Diagnosis for tccp using a Linear Temporal Logic*
}

\author{
MARCO COMINI, LAURA TITOLO \\ DIMI, Università degli Studi di Udine, Italy \\ \{marco.comini, laura.titolo\}@uniud.it \\ ALICIA VILLANUEVA ${ }^{\dagger}$ \\ DSIC, Universitat Politècnica de València, Spain \\ villanue@dsic.upv.es
}

\begin{abstract}
Automatic techniques for program verification usually suffer the wellknown state explosion problem. Most of the classical approaches are based on browsing the structure of some form of model (which represents the behavior of the program) to check if a given specification is valid. This implies that a part of the model has to be built, and sometimes the needed fragment is quite huge.

In this work, we provide an alternative automatic decision method to check whether a given property, specified in a linear temporal logic, is valid w.r.t. a tccp program. Our proposal (based on abstract interpretation techniques) does not require to build any model at all. Our results guarantee correctness but, as usual when using an abstract semantics, completeness is lost.
\end{abstract}

Key Words: concurrent constraint paradigm, linear temporal logic, abstract diagnosis, decision procedures, program verification

\section{Introduction}

The Concurrent Constraint Paradigm (ccp, [20]) is a simple, logic model which is different from other (concurrent) programming paradigms mainly due to the notion of store-as-constraint that replaces the classical store-as-valuation model. Within this family, [9] introduced the Timed Concurrent Constraint Language (tccp in short) by adding to the original $c c p$ model the notion of time and the

\footnotetext{
${ }^{*}$ This work has been published in Theory and Practice of Logic Programming, volume 14, pages 787-801, 2014. Cambridge University Press.

${ }^{\dagger}$ This work has been partially supported by the EU (FEDER) and the Spanish MEC/MICINN, ref. TIN 2010-21062-C02-0, and by Generalitat Valenciana, ref. PROMETEO2011/052.
} 
ability to capture the absence of information. With these features, one can specify behaviors typical of reactive systems such as timeouts or preemption actions. It is well-known that verifying concurrent systems by hand can be an extremely hard task. Thus, the development of automatic formal methods is essential. One of the most known automatic techniques for formal verification is model checking $[4,19]$. It consists in an exhaustive analysis of a finite-state system to check the satisfaction of a given property; thus the state-explosion problem is its main drawback and, for this reason, many proposals in the literature try to mitigate it.

All the proposals of model checking have in common that a part of the model of the (target) program has to be built, and sometimes the needed fragment is quite huge. In this work, we propose a completely different approach to the formal verification of temporal (LTL) properties of concurrent (reactive) systems specified in $t c c p$. We formalize a method to validate a specification of the expected behavior of a tccp program $P$, expressed by a linear temporal formula $\phi$, which does not require to build any model at all.

The linear temporal logic we use to express specifications, csLTL, is an adaptation of the propositional LTL logic to the concurrent constraint framework. This logic is also used as the basis of the abstract domain for a new (abstract) semantics for the language.

In brief, our method is an extension of abstract diagnosis for tccp [5] where the abstract domain $\mathbb{F}$ is formed by csLTL formulas. We cannot use the original abstract diagnosis framework of [5] since $\mathbb{F}$ is not a complete lattice.

The contributions of this work are the following:

- A new abstract semantics for $t c c p$ programs based on csLTL formulas;

- A novel and effective method to validate csLTL properties based on the ideas of abstract diagnosis. This proposal intuitively consists in viewing $P$ as a formula transformer by means of an (abstract) immediate consequence operator $\hat{\mathcal{D}} \llbracket P \rrbracket$ which works on csLTL formulas. Then, to decide the validity of $\phi$, we just have to check if $\hat{\mathcal{D}} \llbracket P \rrbracket_{\phi}$ (i.e., the $P$-transformation of $\phi$ ) implies $\phi$;

- An automatic decision procedure for csLTL properties that makes our method effective.

With our technique we can check, for instance, that, at a railway crossing system, each time a train is approaching, the gate is down, or that whenever a train has crossed, the gate is up. When a property is non valid, the method identifies the buggy process declaration. Technical results of Sections 3 and 4 can be found in [8]. 


\section{The small-step operational behavior of the tccp language}

The tccp language [9] is particularly suitable to specify reactive and time critical systems. As the other languages of the $c c p$ paradigm [21], it is parametric w.r.t. a cylindric constraint system which handles the data information of the program in terms of constraints. The computation progresses as the concurrent and asynchronous activity of several agents that can accumulate information in a store, or query information from it. Briefly, a cylindric constraint system ${ }^{1}$ is an algebraic structure $\mathbf{C}=\langle\mathcal{C}, \leq, \otimes$, false, true, Var, $\exists\rangle$ composed of a set of constraints $\mathcal{C}$ such that $(\mathcal{C}, \leq)$ is a complete algebraic lattice where $\otimes$ is the lub operator and false and true are respectively the greatest and the least element of $\mathcal{C}$; Var is a denumerable set of variables and $\exists$ existentially quantifies variables over constraints. The entailment $\vdash$ is the inverse of $\leq$.

Given a cylindric constraint system $\mathbf{C}$ and a set of process symbols $\Pi$, the syntax of agents is given by the grammar:

$$
A::=\operatorname{skip}|\operatorname{tell}(c)| A \| A|\exists x A| \sum_{i=1}^{n} \operatorname{ask}\left(c_{i}\right) \rightarrow A \mid \text { now } c \text { then } A \text { else } A \mid p(\vec{x})
$$

where $c, c_{1}, \ldots, c_{n}$ are finite constraints in $\mathbf{C} ; p_{/ m} \in \Pi$ and $\vec{x}$ denotes a generic tuple of $m$ variables. A tccp program is an object of the form $D$. A, where $A$ is an agent, called initial agent, and $D$ is a set of process declarations of the form $p(\vec{x})$ :- $A$ (for some agent $A$ ). The notion of time is introduced by defining a discrete and global clock.

The operational semantics of tccp, defined in [9], is formally described by a transition system $T=($ Conf,$\rightarrow)$. Configurations in Conf are pairs $\langle A, c\rangle$ representing the agent $A$ to be executed in the current global store $c$. The transition relation $\rightarrow \subseteq$ Conf $\times$ Conf is the least relation satisfying the rules of Figure 1. Each transition step takes exactly one time-unit.

Example 2.1 (Guiding example) Through the paper, we use as guiding example a part of the full specification of a railway crossing system introduced in [3]. Let us call $D_{m}$ the following $t c c p$ declaration:

$$
\begin{aligned}
& \operatorname{master}(C, G):-\exists C^{\prime}, G^{\prime}(\text { now }(C=[\text { near } \mid \text { _ }]) \text { then } \\
& \text { tell }\left(C=\left[\text { near } \mid C^{\prime}\right]\right) \| \operatorname{tell}\left(G=\left[\text { down } \mid G^{\prime}\right]\right) \| \operatorname{master}\left(C^{\prime}, G^{\prime}\right) \\
& \text { else now }(C=[\text { out } \mid-]) \text { then } \\
& \text { tell }\left(C=\left[\text { out } \mid C^{\prime}\right]\right) \| \text { tell }\left(G=\left[u p \mid G^{\prime}\right]\right) \| \operatorname{master}\left(C^{\prime}, G^{\prime}\right) \\
& \text { else master }(C, G))
\end{aligned}
$$

Due to the monotonicity of the store, streams (written in a list-fashion way) are used to model imperative-style variables [9]. The master process uses an input channel $C$ (implemented as a stream) through which it receives signals from the

\footnotetext{
${ }^{1}$ See $[9,21]$ for more details on cylindric constraint systems.

${ }^{2}$ The auxiliary agent $\exists^{l} x A$ makes explicit the local store $l$ of $A$. This auxiliary agent is linked to the principal hiding construct by setting the initial local store to true, thus $\exists x A:=\exists^{\text {true }} x A$.
} 


$$
\begin{aligned}
& \overline{\langle\operatorname{tell}(c), d\rangle \rightarrow\langle\text { skip, } c \otimes d\rangle} d \neq \text { false } \\
& \overline{\left\langle\sum_{i=1}^{n} \operatorname{ask}\left(c_{i}\right) \rightarrow A_{i}, d\right\rangle \rightarrow\left\langle A_{j}, d\right\rangle} j \in[1, n], d \vdash c_{j}, d \neq \text { false } \\
& \frac{\langle A, d\rangle \rightarrow\left\langle A^{\prime}, d^{\prime}\right\rangle}{\langle\text { now } c \text { then } A \text { else } B, d\rangle \rightarrow\left\langle A^{\prime}, d^{\prime}\right\rangle} d \vdash c \\
& \frac{\langle A, d\rangle \nrightarrow}{\langle\text { now } c \text { then } A \text { else } B, d\rangle \rightarrow\langle A, d\rangle} d \vdash c, d \neq \text { false } \\
& \frac{\langle B, d\rangle \rightarrow\left\langle B^{\prime}, d^{\prime}\right\rangle}{\langle\text { now } c \text { then } A \text { else } B, d\rangle \rightarrow\left\langle B^{\prime}, d^{\prime}\right\rangle} d \nLeftarrow c \\
& \frac{\langle B, d\rangle+}{\langle\text { now } c \text { then } A \text { else } B, d\rangle \rightarrow\langle B, d\rangle} d \forall c \\
& \frac{\langle A, d\rangle \rightarrow\left\langle A^{\prime}, d^{\prime}\right\rangle\langle B, d\rangle \rightarrow\left\langle B^{\prime}, c^{\prime}\right\rangle}{\langle A \| B, d\rangle \rightarrow\left\langle A^{\prime} \| B^{\prime}, d^{\prime} \otimes c^{\prime}\right\rangle} \\
& \frac{\langle A, d\rangle \rightarrow\left\langle A^{\prime}, d^{\prime}\right\rangle\langle B, d\rangle \rightarrow}{\langle A \| B, d\rangle \rightarrow\left\langle A^{\prime} \| B, d^{\prime}\right\rangle} \\
& \langle B \| A, d\rangle \rightarrow\left\langle B \| A^{\prime}, d^{\prime}\right\rangle \\
& \frac{\left\langle A, l \otimes \exists_{x} d\right\rangle \rightarrow\left\langle B, l^{\prime}\right\rangle}{\left\langle\exists^{l} x A, d\right\rangle \rightarrow\left\langle\exists^{l^{\prime}} x B, d \otimes \exists_{x} l^{\prime}\right\rangle}
\end{aligned}
$$

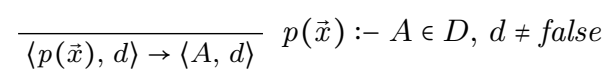

Figure 1: The transition system for $t c c p .^{2}$

environment (trains), and an output channel $G$ through which it sends orders to a gate process. It checks the input channel for a near signal (the guard in the first now agent), in which case it sends (tells) the order down through $G$, links the future values $\left(C^{\prime}\right)$ of the stream $C$ and restarts the check at the following time instant (recursive call master $\left(C^{\prime}, G^{\prime}\right)$ ). If the near signal is not detected, then, the else branch looks for the out signal and (if present) behaves dually to the first branch. Finally, if no signal is detected at the current time instant (last else branch), then the process keeps checking from the following time instant.

In this work, we prove the correctness of our technique w.r.t. the denotational concrete semantics of [6], which is fully-abstract (correct and complete) w.r.t. the small-step operational behavior of tccp. Also csLTL is interpreted over this denotational model. We thus introduce the most relevant aspects of such semantics.

The denotational semantics of a tccp program consists of a set of conditional (timed) traces that represent, in a compact way, all the possible behaviors that the program can manifest when fed with an input (initial store). Conditional traces can be seen as hypothetical computations in which, for each time instant, we have a condition representing the information that the global store has to 
satisfy in order to proceed to the next time instant. Briefly, a conditional trace is a (possibly infinite) sequence $t_{1} \cdots t_{n} \cdots$ of conditional states, which can be of three forms:

conditional store: a pair $\eta \nrightarrow c$, where $\eta$ is a condition and $c \in \mathbf{C}$ a store;

stuttering: the construct $\operatorname{stutt}(C)$, with $C \subseteq \mathbf{C} \backslash\{$ true $\}$;

end of a process: the construct $\otimes$.

Intuitively, the conditional store $\eta \nrightarrow c$ means that, provided condition $\eta$ is satisfied by the current store, the computation proceeds so that in the following time instant, the store is $c$. A condition $\eta$ is a pair $\eta=\left(\eta^{+}, \eta^{-}\right)$where $\eta^{+} \in \mathbf{C}$ and $\eta^{-} \in \wp(\mathbf{C})$ are called positive and negative condition, respectively. The positive/negative condition represents information that a given store must/must not entail, thus they have to be consistent in the sense that $\forall c^{-} \in \eta^{-} \eta^{+} \forall c^{-}$. The stuttering construct models the suspension of the computation when none of the guards in a non-deterministic agent is satisfied. $C$ is the set of guards in the non-deterministic agent. Conditional traces are monotone (i.e., for each $t_{i}=\eta_{i} \nrightarrow c_{i}$ and $t_{j}=\eta_{j} \nrightarrow c_{j}$ such that $j \geq i, c_{j} \vdash c_{i}$ ) and consistent (i.e., each store in a trace does not entail the negative conditions of the following conditional state).

We denote the domain of conditional trace sets as $\mathbb{M}$. ( $\mathbb{M}, \subseteq, \sqcup, \sqcap, \mathbf{M},\{\epsilon\})$ is a complete lattice, where $M_{1} \sqsubseteq M_{2} \Leftrightarrow \forall r_{1} \in M_{1} \exists r_{2} \in M_{2} . r_{1}$ is a prefix of $r_{2}$. We define as $\bar{\exists}_{x} r$ the sequence resulting by removing from $r \in \mathbf{M}$ all the information about the variable $x$. We distinguish two special classes of conditional traces. $r \in \mathbf{M}$ is said to be self-sufficient if the first condition is (true, $\varnothing$ ) and, for each $t_{i}=\left(\eta_{i}^{+}, \eta_{i}^{-}\right) \nrightarrow c_{i}$ and $t_{i+1}=\left(\eta_{i+1}^{+}, \eta_{i+1}^{-}\right) \nrightarrow c_{i+1}, c_{i} \vdash \eta_{i+1}^{+}$(each store satisfies the successive condition). Moreover, $r$ is $x$-self-sufficient if $\bar{\exists}_{\operatorname{Var} \backslash\{x\}} r$ is self-sufficient. Thus, this definition demands that for self-sufficient conditional traces, no additional information (from other agents) is needed in order to complete the computation. ${ }^{3}$

The semantics definition is based on a semantics evaluation function $\mathcal{A} \llbracket A \rrbracket_{\mathcal{I}}$ [6] which, given an agent $A$ and an interpretation $\mathcal{I}$, builds the conditional traces associated to $A$. The interpretation $\mathcal{I}$ is a function which associates to each process symbol a set of conditional traces "modulo variance". The semantics for a set of process declarations $D$ is the fixpoint $\mathcal{F} \llbracket D \rrbracket:=$ lfp $(\mathcal{D} \llbracket D \rrbracket)$ of the continuous immediate consequences operator $\mathcal{D} \llbracket D \rrbracket_{\mathcal{I}}(p(\vec{x})):=\bigsqcup_{p(\vec{x}):-A \in D} \mathcal{A} \llbracket A \rrbracket_{\mathcal{I}}$. Proof of full abstraction w.r.t. the operational behavior of tccp is given in [6].

Example 2.2 Consider the process declaration $D_{m}$ of Example 2.1. Given an interpretation $\mathcal{I}$, the semantics of master $(C, G)$ is graphically represented in Figure 2, where we have used some shortcuts for characteristic constraints. Namely, $c_{\text {near }}:=\left(C=\left[\right.\right.$ near $\left.\left.\mid{ }_{-}\right]\right), c_{\text {near }}^{\prime}:=\exists_{C^{\prime}}\left(C=\left[\right.\right.$ near $\left.\left.\mid C^{\prime}\right]\right), c_{\text {down }}:=\exists_{G^{\prime}}(G=$ $\left[\right.$ down $\left.\left.\mid G^{\prime}\right]\right), c_{\text {out }}:=(C=[$ out $\mid-]), c_{\text {out }}^{\prime}:=\exists_{C^{\prime}}\left(C=\left[\right.\right.$ out $\left.\left.\mid C^{\prime}\right]\right), c_{\text {up }}:=\exists_{G^{\prime}}(G=$ $\left.\left[u p \mid G^{\prime}\right]\right)$.

\footnotetext{
${ }^{3}$ The set of all self-sufficient conditional traces can be considered as a generalization (using conditional states in place of stores) of the traditional strongest postcondition for semantics.
} 


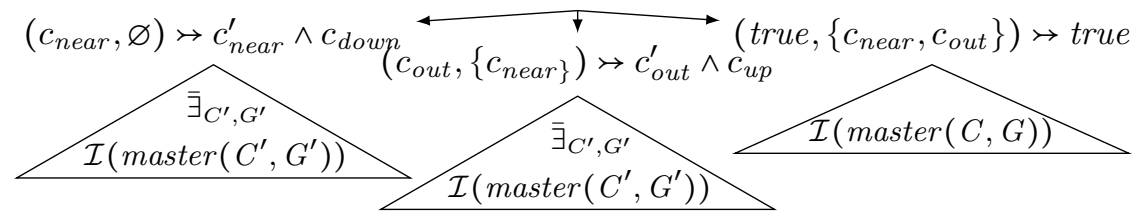

Figure 2: Tree representation of $\mathcal{D} \llbracket\left\{D_{m}\right\} \rrbracket_{\mathcal{I}}($ master $(C, G))$ of Example 2.2.

The branch on the left represents the computation when a near signal arrives. The first conditional state requires that $c_{\text {near }}$ holds, thus the constraints $c_{\text {near }}^{\prime}$ and $c_{\text {down }}$ are concurrently added to the store during that computational step. A recursive call is also concurrently invoked. Process calls do not modify the store when invoked, but they affect the store from the following time instant, which is graphically represented by the triangle labeled with the interpretation of the process. The branch in the middle is taken only if $c_{\text {out }}$ is entailed and $c_{\text {near }}$ is not entailed by the initial store (it occurs in the negative condition of the first conditional state in that branch). Finally, the branch on the right represents the case when both $c_{\text {near }}$ and $c_{\text {out }}$ are not entailed by the initial store.

\section{Abstract semantics for tccp over csLTL formu- las}

In this section, we present a novel abstract semantics over formulas that approximates the small-step semantics described in Section 2 and, therefore, the small-step operational behavior of a tccp program. To this end, we first define an abstract domain of logic formulas which is a variation of the classical Linear Temporal Logic [17]. Following [18, 10, 11, 22], the idea is to replace atomic propositions by constraints of the underlying constraint system.

Definition 3.1 (csLTL formulas) Given a cylindric constraint system $\mathbf{C}, c \epsilon$ $\mathbf{C}$ and $x \in$ Var, formulas of the Constraint System Linear Temporal Logic over C are:

$$
\phi::=\text { true } \mid \text { false }|c| \dot{\sim} \phi|\phi \dot{\wedge} \phi| \dot{\exists}_{x} \phi|\bigcirc \phi| \phi \mathcal{U} \phi .
$$

$\operatorname{cs} T L_{\mathbf{C}}$ is the set of all temporal formulas over $\mathbf{C}$ (we omit $\mathbf{C}$ if clear from the context).

true, false, $\dot{\neg}, \dot{\wedge}, \bigcirc \phi, \phi_{1} \mathcal{U} \phi_{2}$ have the classical logical meaning. The atomic formula $c \in \mathbf{C}$ states that $c$ has to be entailed by the current store. $\dot{\exists}_{x} \phi$ is the existential quantification over the set of variables Var. As usual, we use

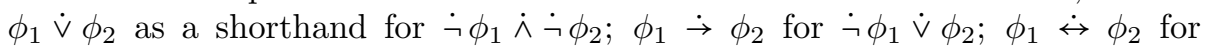
$\phi_{1} \dot{\rightarrow} \phi_{2} \dot{\wedge} \phi_{2} \dot{\rightarrow} \phi_{1} ; \diamond \phi$ for true $\mathcal{U} \phi$ and $\square \phi$ for $\dot{\neg} \diamond \dot{\neg} \phi$. A constraint formula is an atomic formula $c$ or its negation $\dot{\neg}$. Formulas $\bigcirc \phi$ and $\dot{\neg} \bigcirc \phi$ are called 
next formulas. Constraint and next formulas are said to be elementary formulas. Finally, formulas of the form $\phi_{1} \mathcal{U} \phi_{2}, \diamond \phi$ or $\dot{\neg}(\square \phi)$ are called eventualities.

We define the abstract domain $\mathbb{F}:=\operatorname{csLTL} / \leftrightarrow$ (i.e., the domain formed by csLTL formulas modulo logical equivalence) ordered by $\dot{\rightarrow}$. The algebraic lattice $(\mathbb{F}, \dot{\rightarrow}, \dot{V}, \dot{\Lambda}$, true, false $)$ is not complete, since both $\dot{\wedge}$ and $\dot{V}$ always exist just for finite sets of formulas.

The semantics of a temporal formula is typically defined in terms of an infinite sequence of states which validates it. Here we use conditional traces instead. As usually done in the context of temporal logics, we define the satisfaction relation $\vDash$ only for infinite conditional traces. We implicitly transform finite traces (which end in $\otimes$ ) by replicating the last store infinite times.

Definition 3.2 The semantics of $\phi \in \mathbb{F}$ is given by function $\gamma^{\mathbb{F}}: \mathbb{F} \rightarrow \mathbb{M}$ defined as $\gamma^{\mathbb{F}}(\phi):=\sqcup\{r \in \mathbf{M} \mid r \vDash \phi\}$, where, for each $\phi, \phi_{1}, \phi_{2} \in \operatorname{csLTL}, c \in \mathbf{C}$ and $r \in \mathbf{M}$, satisfaction relation $\vDash$ is defined as:

$$
\begin{aligned}
& r \vDash \text { true and } r \neq \text { false } \\
& \left(\eta^{+}, \eta^{-}\right) \nrightarrow d \cdot r^{\prime} \vDash c \quad \text { iff } \eta^{+} \vdash c \\
& \operatorname{stutt}\left(\eta^{-}\right) \cdot r^{\prime} \vDash c \quad \text { iff } \forall d^{-} \in \eta^{-} . c H d^{-} \text {and } r^{\prime} \vDash c \\
& r \vDash \dot{\sim} \phi \\
& \text { iff } r \notin \phi \\
& r \vDash \phi_{1} \dot{\wedge} \phi_{2} \quad \text { iff } r \vDash \phi_{1} \text { and } r \vDash \phi_{2} \\
& r \vDash \bigcirc \phi \quad \text { iff } r^{1} \vDash \phi^{4} \\
& r \vDash \phi_{1} \mathcal{U} \phi_{2} \quad \text { iff } \exists i \geq 1 . \forall j<i . r^{i} \vDash \phi_{2} \text { and } r^{j} \vDash \phi_{1} \\
& r \vDash \dot{\exists}_{x} \phi \quad \text { iff exists } r^{\prime} \text { s.t. } \bar{\exists}_{x} r^{\prime}=\bar{\exists}_{x} r, r^{\prime} x \text {-self-sufficient and } r^{\prime} \vDash \phi
\end{aligned}
$$

We say that $\phi \in \mathbb{F}$ is a sound approximation of $R \in \mathbb{M}$ if $R \sqsubseteq \gamma^{\mathbb{F}}(\phi)$. $\phi$ is said to be satisfiable if there exists $r \in \mathbf{M}$ such that $r \vDash \phi$, while it is valid if, for all $r \in \mathbf{M}, r \vDash \phi$.

All the cases are fairly standard except (3.1b) and (3.1c). The conditional trace $r=\left(\eta^{+}, \eta^{-}\right) \rightarrow d \cdot r^{\prime}$ prescribes that $\eta^{+}$is entailed by the current store, thus $r$ models all the constraint formulas $c$ such that $\eta^{+} \vdash c$. We have to note that, by the monotonicity of the store of tccp computations, the positive conditions in conditional traces contains all the information previously added in the constraint store. Furthermore, by the definition of condition, since $\eta^{+}$ cannot be in contradiction with $\eta^{-}$, it holds that neither $c$ is in contradiction with $\eta^{-}$. Thus, the conditional trace $\operatorname{stutt}\left(\eta^{-}\right) \cdot r^{\prime}$ models all the constraint formulas $c$ that are not in contradiction with the set $\eta^{-}$and such that $c$ holds in the continuation $r^{\prime}$ by monotonicity.

Lemma 3.3 The function $\gamma^{\mathbb{F}}$ is monotonic, injective and $\sqcap$-distributive.

\section{1 csLTL Abstract Semantics}

The technical core of our semantics definition is the csLTL agent semantics evaluation function $\hat{\mathcal{A}} \llbracket A \rrbracket$ which, given an agent $A$ and an interpretation $\hat{\mathcal{I}}$ (for the

\footnotetext{
${ }^{4} r^{k}$ denotes the sub-sequence of $r$ starting from state $k$.
} 
process symbols of $A$ ), builds a csLTL formula which is a sound approximation of the (concrete) behavior of $A$. In the sequel, we denote by $\mathbb{A}_{\mathbf{C}}^{\Pi}$ the set of agents and $\mathbb{D}_{\mathbf{C}}^{\Pi}$ the set of sets of process declarations built on signature $\Pi$ and constraint system $\mathbf{C}$.

Definition 3.4 Let $\mathbb{P} \mathbb{C}:=\{p(\vec{x}) \mid p \in \Pi, \vec{x}$ are distinct variables $\}$. An $\mathbb{F}$ interpretation is a function $\mathbb{P C} \rightarrow \mathbb{F}$ modulo variance ${ }^{5}$. Two functions $I, J: \mathbb{P C} \rightarrow$ $\mathbb{F}$ are variants if for each $\pi \in \mathbb{P C}$ there exists a renaming $\rho$ such that $(I \pi) \rho=$ $J(\pi \rho)$. The semantic domain $\mathbb{I}_{\mathbb{F}}$ is the set of all $\mathbb{F}$-interpretations ordered by the point-wise extension of $\rightarrow$.

Definition 3.5 (csLTL Semantics) Given $A \in \mathbb{A}_{\mathbf{C}}^{\Pi}$ and $\hat{\mathcal{I}} \in \mathbb{I}_{\mathbb{F}}$, we define the csLTL semantics evaluation $\hat{\mathcal{A}} \llbracket A \rrbracket_{\hat{\mathcal{I}}}$ by structural induction as follows.

$$
\begin{aligned}
& \hat{\mathcal{A}} \llbracket \operatorname{skip} \rrbracket_{\hat{\mathcal{I}}}:=\text { true } \\
& \hat{\mathcal{A}} \llbracket A_{1} \| A_{2} \rrbracket_{\hat{\mathcal{I}}}:=\hat{\mathcal{A}} \llbracket A_{1} \rrbracket_{\hat{\mathcal{I}}} \dot{\wedge} \hat{\mathcal{A}} \llbracket A_{2} \rrbracket_{\hat{\mathcal{I}}} \\
& \hat{\mathcal{A}} \llbracket \operatorname{tell}(c) \rrbracket_{\hat{\mathcal{I}}}:=\bigcirc c \\
& \hat{\mathcal{A}} \llbracket \exists x \rrbracket_{\hat{\mathcal{I}}}:=\dot{\exists} x \hat{\mathcal{A}} \llbracket A \rrbracket_{\hat{\mathcal{I}}} \\
& \hat{\mathcal{A}} \llbracket p(\vec{x}) \rrbracket_{\hat{\mathcal{I}}}:=\bigcirc \hat{\mathcal{I}}(p(\vec{x})) \\
& \hat{\mathcal{A}} \llbracket \sum_{i=1}^{n} \operatorname{ask}\left(c_{i}\right) \rightarrow A_{i} \rrbracket_{\hat{\mathcal{I}}}:=\square\left(\dot{\Lambda}_{i=1}^{n} \dot{\neg} c_{i}\right) \dot{\vee}\left(\left(\dot{\Lambda}_{i=1}^{n} \dot{\neg} c_{i}\right) \mathcal{U} \dot{\vee}_{i=1}^{n}\left(c_{i} \dot{\wedge} \bigcirc \hat{\mathcal{A}} \llbracket A_{i} \rrbracket_{\hat{\mathcal{I}}}\right)\right) \\
& \hat{\mathcal{A}} \llbracket \text { now } c \text { then } A_{1} \text { else } A_{2} \rrbracket_{\hat{\mathcal{I}}}:=\left(c \dot{\wedge} \hat{\mathcal{A}} \llbracket A_{1} \rrbracket_{\hat{\mathcal{I}}}\right) \dot{\vee}\left(\dot{\neg} c \dot{\wedge} \hat{\mathcal{A}} \llbracket A_{2} \rrbracket_{\hat{\mathcal{I}}}\right)
\end{aligned}
$$

Given $D \in \mathbb{D}_{\mathbf{C}}^{\Pi}$ we define the immediate consequence operator $\hat{\mathcal{D}} \llbracket D \rrbracket: \mathbb{I}_{\mathbb{F}} \rightarrow \mathbb{I}_{\mathbb{F}}$ as

$$
\hat{\mathcal{D}} \llbracket D \rrbracket_{\hat{\mathcal{I}}}(p(\vec{x})):=\dot{\bigvee}\left\{\hat{\mathcal{A}} \llbracket A \rrbracket_{\hat{\mathcal{I}}} \mid p(\vec{x}):-A \in D\right\}
$$

We have that $\hat{\mathcal{A}}$ is a sound approximation of $\mathcal{A}$ and $\hat{\mathcal{D}}$ is a sound approximation of $\mathcal{D}$.

Theorem 3.6 (Correctness of $\hat{\mathcal{A}}$ and $\hat{\mathcal{D}}$ ) Let $A \in \mathbb{A}_{\mathbf{C}}^{\Pi}, D \in \mathbb{D}_{\mathbf{C}}^{\Pi}$ and $\hat{\mathcal{I}} \in \mathbb{I}_{\mathbb{F}}$. Then, $\mathcal{A} \llbracket A \rrbracket_{\gamma^{\mathbb{F}}(\hat{\mathcal{I}})} \sqsubseteq \gamma^{\mathbb{F}}\left(\hat{\mathcal{A}} \llbracket A \rrbracket_{\hat{\mathcal{I}}}\right)$ and $\mathcal{D} \llbracket D \rrbracket_{\gamma^{\mathbb{F}}(\hat{\mathcal{I}})} \sqsubseteq \gamma^{\mathbb{F}}\left(\hat{\mathcal{D}} \llbracket D \rrbracket_{\hat{\mathcal{I}}}\right)$.

Example 3.7 Consider the process declaration $D_{m}$ of Example 2.1 and let us use $\bigcirc^{n}$ to abbreviate the repetition of $\bigcirc n$-times. Given $\hat{\mathcal{I}} \in \mathbb{I}_{\mathbb{F}}$, with Definition 3.5 we compute

$$
\left.\phi_{M}(\hat{\mathcal{I}}):=\hat{\mathcal{D}} \llbracket\left\{D_{m}\right\} \rrbracket_{\hat{\mathcal{I}}}(\text { master }(C, G))\right)=\phi_{\text {near }}(\hat{\mathcal{I}}) \dot{\vee} \phi_{\text {out }}(\hat{\mathcal{I}}) \dot{\vee} \phi_{\text {cwait }}(\hat{\mathcal{I}})
$$

where

$$
\begin{gathered}
\phi_{\text {near }}(\hat{\mathcal{I}})=\dot{\exists}_{C^{\prime}, G^{\prime}}\left(C=\left[\text { near } \mid \_\right] \dot{\wedge} \bigcirc C=\left[\text { near } \mid C^{\prime}\right] \dot{\wedge} \bigcirc G=\left[\text { down } \mid G^{\prime}\right] \dot{\wedge} \bigcirc \hat{\mathcal{I}}\left(\text { master }\left(C^{\prime}, G^{\prime}\right)\right)\right) \\
\phi_{\text {out }}(\hat{\mathcal{I}})=\dot{\exists}_{C^{\prime}, G^{\prime}}\left(\dot{\mathcal{\imath}}(C=[\text { near } \mid \text { _ }]) \dot{\wedge} \bigcirc C=\left[\text { out } \mid C^{\prime}\right] \dot{\wedge}\right. \\
\left.C=[\text { out } \mid \text { _ }] \dot{\wedge} \bigcirc G=\left[\text { up } \mid G^{\prime}\right] \dot{\wedge} \bigcirc \hat{\mathcal{I}}\left(\operatorname{master}\left(C^{\prime}, G^{\prime}\right)\right)\right)
\end{gathered}
$$

\footnotetext{
${ }^{5}$ i.e., a family of elements of $\mathbb{F}$, indexed by $\mathbb{P} \mathbb{C}$, modulo variance.
} 


$$
\phi_{\text {cwait }}(\hat{\mathcal{I}})=\dot{\neg}\left(C=\left[\text { near }\left.\right|_{-}\right]\right) \dot{\wedge} \dot{\neg}\left(C=\left[\text { out }\left.\right|_{-}\right]\right) \dot{\wedge} \bigcirc \hat{\mathcal{I}}(\text { master }(C, G))
$$

The three disjuncts of $\phi_{M}(\hat{\mathcal{I}})$ match the three possible behaviors of master $(C, G)$ : when signal near is emitted by the train, when out is emitted, and when no signal arrives.

\section{Abstract diagnosis of tccp with csLTL formu- las}

Since $\mathbb{F}$ is not a complete lattice, it is impossible to find for the function $\gamma^{\mathbb{F}}$ an adjoint function $\alpha$ which forms a Galois Connection $\langle\alpha, \gamma\rangle$, and therefore we cannot use the abstract diagnosis framework for tccp defined in [5]. Thus, we propose in this section a new weaker version of abstract diagnosis that works on $\mathbb{F}^{6}$.

Given a set of declarations $D$ and $\hat{\mathcal{S}} \in \mathbb{I}_{\mathbb{F}}$, which is the specification of the abstract intended behavior of $D$ over $\mathbb{F}$, we say that

1. $D$ is (abstractly) partially correct w.r.t. $\hat{\mathcal{S}}$ if $\mathcal{F} \llbracket D \rrbracket \sqsubseteq \gamma^{\mathbb{F}}(\hat{\mathcal{S}})$.

2. $D$ is (abstractly) complete w.r.t. $\hat{\mathcal{S}}$ if $\gamma^{\mathbb{F}}(\hat{\mathcal{S}}) \sqsubseteq \mathcal{F} \llbracket D \rrbracket$.

The differences between $\mathcal{F} \llbracket D \rrbracket$ and $\gamma^{\mathbb{F}}(\hat{\mathcal{S}})$ are usually called symptoms. Many of the symptoms are just a consequence of some "originating" ones, those which are the direct consequence of errors. The abstract diagnosis determines exactly the "originating" symptoms and, in the case of incorrectness, the faulty process declarations in $D$. This is captured by the definitions of abstractly incorrect process declaration and abstract uncovered element: ${ }^{7}$

Definition 4.1 Let $D \in \mathbb{D}_{\mathbf{C}}^{\Pi}, R$ a process declaration for process $p, \phi_{t} \in \mathbb{F}$ and $\hat{\mathcal{S}} \in \mathbb{I}_{\mathbb{F}}$.

- $R$ is abstractly incorrect w.r.t. $\hat{\mathcal{S}}$ (on testimony $\left.\phi_{t}\right)$ if $\phi_{t} \dot{\rightarrow} \hat{\mathcal{D}} \llbracket\{R\} \rrbracket_{\hat{\mathcal{S}}}(p(\vec{x}))$ and $\phi_{t} \dot{\wedge} \hat{\mathcal{S}}(p(\vec{x}))=$ false.

- $\phi_{t}$ is an uncovered element for $p(\vec{x})$ w.r.t. $\hat{\mathcal{S}}$ if $\phi_{t} \dot{\rightarrow} \hat{\mathcal{S}}(p(\vec{x}))$ and $\phi_{t} \dot{\wedge} \hat{\mathcal{D}} \llbracket D \rrbracket_{\hat{\mathcal{S}}}(p(\vec{x}))=$ false.

Informally, $R$ is abstractly incorrect if it derives a wrong abstract element $\phi_{t}$ from the intended semantics. Dually, $\phi_{t}$ is uncovered if the declarations cannot derive it from the intended semantics.

\footnotetext{
${ }^{6}$ Actually, the proposal is defined using just $\gamma^{\mathbb{F}}$ only for the sake of simplicity. It could easily be defined parametrically w.r.t. a suitable family of concretization functions.

${ }^{7}$ It is worth noticing that although the notions defined in this section are similar to those defined for the standard approach, the formal definitions and proofs are different due to the weaker framework.
} 
Theorem 4.2 Let $D \in \mathbb{D}_{\mathbf{C}}^{\Pi}$ and $\hat{\mathcal{S}} \in \mathbb{I}_{\mathbb{F}}$. (1) If there are no abstractly incorrect process declarations in $D$ (i.e., $\hat{\mathcal{D}} \llbracket D \rrbracket_{\hat{\mathcal{S}}} \rightarrow \hat{\mathcal{S}}$ ), then $D$ is partially correct w.r.t. $\hat{\mathcal{S}}$. (2) If $D$ is partially correct w.r.t. $\hat{\mathcal{S}}$ and $D$ has abstract uncovered elements then $D$ is not complete.

Absence of abstractly incorrect declarations is a sufficient condition for partial correctness, but it is not necessary. Because of the approximation, it can happen that a (concretely) correct declaration is abstractly incorrect. Hence, abstract incorrect declarations are in general just a warning about a possible source of errors. However, an abstract correct declaration cannot contain an error; thus, no (manual) inspection is needed for declarations which are not abstractly incorrect. Moreover, as shown by the following theorem, all concrete errorsthat are "visible" - are indeed detected, as they lead to an abstract incorrectness or abstract uncovered. Intuitively, a concrete error is visible if we can express a formula $\phi$ whose concretization reveals the error (i.e., if the logic is expressive enough).

Theorem 4.3 Let $R$ be a process declaration for $p(\vec{x}), \mathcal{S}$ a concrete specification and $\hat{\mathcal{S}}$ a sound approximation for $\mathcal{S}$ (i.e., $\left.\mathcal{S} \subseteq \gamma^{\mathbb{F}}(\hat{\mathcal{S}})\right)$. (1) If $\mathcal{D} \llbracket\{R\} \rrbracket_{\mathcal{S}}$ 车 $\gamma^{\mathbb{F}}(\hat{\mathcal{S}})$ and it exists $\phi_{t}$ such that $\gamma^{\mathbb{F}}\left(\phi_{t}\right) \sqsubseteq \mathcal{D} \llbracket\{R\} \rrbracket_{\mathcal{S}}(p(\vec{x}))$ and $\phi_{t} \dot{\wedge} \mathcal{\mathcal { S }}(p(\vec{x}))=$ false, then $R$ is abstractly incorrect w.r.t. $\mathcal{S}$ (on testimony $\phi_{t}$ ). (2) If there exists an abstract uncovered element $\phi$ w.r.t. $\hat{\mathcal{S}}$, then there exists $r \in \gamma^{\mathbb{F}}(\phi)$ such that $r \notin \mathcal{D} \llbracket\{R\} \rrbracket_{\mathcal{S}}(p(\vec{x}))$.

Point 2 says that the concrete error has an abstract symptom which is not hidden by the approximation on $\hat{\mathcal{S}}$ and, moreover, there exists a formula $\phi_{t}$ which can express it.

In the following examples, we borrow from [3] the notation for last entailed value of a stream: $X \doteq c$ holds if the last instantiated value in the stream $X$ is $c$.

Example 4.4 We verify (for Example 2.1) that each time a near signal arrives from a train, the order down is sent to a gate process. ${ }^{8}$ To model this property, we define the specification (of the property) $\hat{\mathcal{S}}_{\text {down }}$ as

$$
\phi_{\text {ordersent }}:=\hat{\mathcal{S}}_{\text {down }}(\operatorname{master}(C, G)):=\square(C \dot{\doteq} \text { near } \dot{\rightarrow} \diamond(G \dot{=} \text { down }))
$$

To check whether the program implies the specification $\left(\hat{\mathcal{D}} \llbracket\left\{D_{m}\right\} \rrbracket_{\hat{\mathcal{S}}_{\text {down }}} \dot{\rightarrow} \hat{\mathcal{S}}_{\text {down }}\right)$ we have to check if $\phi_{M}\left(\hat{\mathcal{S}}_{\text {down }}\right) \dot{\rightarrow} \phi_{\text {ordersent }}$ (where $\phi_{M}(\cdot)$ is defined in Example 3.7). Each of the three disjuncts of $\phi_{M}\left(\hat{\mathcal{S}}_{\text {down }}\right)$ implies $\phi_{\text {ordersent }}$. Thus, by Theorem $4.2, D_{m}$ is partially correct w.r.t. $\hat{\mathcal{S}}_{\text {down }}$.

When the check of a process declaration $R$ against a specification $S$ fails, our method reports that $R$ is not partially correct w.r.t. $S$. If this occurs, the formula testimony for the possible incorrectness gives useful information to fix the process declaration or check whether it corresponds to a false positive.

\footnotetext{
${ }^{8} \mathrm{~A}$ more interesting property, namely that, in addition, the gate is eventually down, is verified in [8]. Here we have simplified the property due to space limitations.
} 
Example 4.5 Now we show how our technique detects an error in a buggy set of declarations. We remove instruction tell $\left(G=\left[u p \mid G^{\prime}\right]\right)$ in the process declaration $D_{m}$ (of Example 2.1). To avoid misunderstandings, we call the modified process master' and let $R$ be the new process declaration.

We aim to verify that the order up is sent whenever the signal out is received:

$$
\phi:=\hat{\mathcal{S}}_{\text {up }}\left(\operatorname{master}^{\prime}(C, G)\right):=\square((C \dot{\doteq} \text { out }) \dot{\rightarrow} \diamond(G \dot{\doteq} \text { up }))
$$

We need to compute the (one step) semantics for the (buggy version of the) process:

$$
\phi^{\prime}:=\hat{\mathcal{D}} \llbracket\{R\} \rrbracket_{\hat{\mathcal{S}}_{\text {up }}}\left(\text { master }^{\prime}(C, G)\right)=\phi_{\text {near }}^{\prime} \dot{\vee} \phi_{\text {out }}^{\prime} \dot{\vee} \phi_{\text {cwait }}^{\prime}
$$

where

$$
\begin{aligned}
\phi_{\text {near }}^{\prime} & :=\dot{\exists}_{C^{\prime}, G^{\prime}}\left(C=[\text { near } \mid-] \dot{\wedge} \bigcirc C=\left[\text { near } \mid C^{\prime}\right] \dot{\wedge} \bigcirc G=\left[\text { down } \mid G^{\prime}\right] \dot{\wedge} \bigcirc \hat{\mathcal{S}}_{\text {up }}\left(\text { master }^{\prime}\left(C^{\prime}, G^{\prime}\right)\right)\right) \\
\phi_{\text {out }}^{\prime} & :=\dot{\exists}_{C^{\prime}, G^{\prime}}\left(\dot{\neg}(C=[\text { near } \mid-]) \dot{\wedge} C=[\text { out } \mid-] \dot{\wedge} \bigcirc\left(C=\left[\text { out } \mid C^{\prime}\right] \dot{\wedge} \bigcirc \hat{\mathcal{S}}_{\text {up }}\left(\text { master }^{\prime}\left(C^{\prime}, G^{\prime}\right)\right)\right)\right) \\
\phi_{\text {cwait }}^{\prime} & :=\dot{\neg}\left(C=\left[\text { near } \mid{ }_{-}\right]\right) \dot{\wedge} \dot{\neg}\left(C=\left[\text { out } \mid \_\right]\right) \dot{\wedge} \bigcirc \hat{\mathcal{S}}_{\text {up }}\left(\text { master }^{\prime}(C, G)\right)
\end{aligned}
$$

We detect an incorrectness of $R$ (in master' process) w.r.t. $\hat{\mathcal{S}}_{\text {up }}$ on testimony $\phi_{\text {out }}^{\prime}$ since $\phi_{\text {out }}^{\prime} \dot{\rightarrow} \phi^{\prime}$ and $\phi_{\text {out }}^{\prime} \dot{\wedge} \phi=$ false. The testimony suggests that on channel $C$ we have out signal but we do not see the corresponding up signal on channel $G$.

Our technique behaves negatively for sets of declarations $D$ where $\hat{\mathcal{D}} \llbracket D \rrbracket$ has more than one fixpoint. This happens with programs with loops that do not produce contributes at all (which are in some sense non meaningful programs). In such situations, we can have that the actual behavior does not model a specification $\hat{\mathcal{S}}$ which is a non-least fixpoint of $\hat{\mathcal{D}} \llbracket D \rrbracket$, but, since $\hat{\mathcal{S}}$ is a fixpoint, we do not detect the abstractly incorrect declaration, as shown by the following example.

Example 4.6 (Pathological cases) Let $D_{p}:=\{q(y):-$ now $y=1$ then $q(y)$ else $q(y)\}$ and $\hat{\mathcal{S}}_{p}(q(y)):=\diamond(y=1)$ be the specification. Then, we compute $\hat{\mathcal{D}} \llbracket D_{p} \rrbracket_{\hat{\mathcal{S}}_{p}}(q(y))=(y=1 \dot{\wedge} \diamond y=1) \dot{\vee}(\dot{\neg} y=1 \dot{\wedge} \diamond y=1)$. We can see that $\hat{\mathcal{D}} \llbracket D_{p_{p}} \rrbracket_{\hat{\mathcal{S}}_{p}} \dot{\rightarrow}$ $\diamond(y=1)$, thus $D_{p}$ is partially correct w.r.t. $\hat{\mathcal{S}}_{p}$. However, $y=1$ is not explicitly added by the process.

Note that, if $\hat{\mathcal{S}}(p(\vec{x}))$ is assumed to hold for each process $p(\vec{x})$ defined in $D$ and $\hat{\mathcal{D}} \llbracket D \rrbracket_{\hat{\mathcal{S}}} \dot{\rightarrow} \hat{\mathcal{S}}$, then $\mathcal{F} \llbracket D \rrbracket$ satisfies $\hat{\mathcal{S}}$.

\subsection{An automatic decision procedure for csLTL}

In order to make our abstract diagnosis approach effective, we have defined an automatic decision procedure to check the validity of the formulas involved in Definition 4.1 (of the form $\psi \rightarrow \phi$ with $\phi=\hat{\mathcal{S}}(p(\vec{x}))$ and $\psi=\hat{\mathcal{D}} \llbracket D \rrbracket_{\hat{\mathcal{S}}}(p(\vec{x}))$ ). We adapt to csLTL the tableau construction for Propositional LTL of $[15,16]$. [7] contains a preliminary version of the method. 
Table 1: $\alpha$ - and $\beta$-formulas rules.

\begin{tabular}{|c|c|c|c|c|c|c|}
\hline & $\alpha$ & $A(\alpha)$ & & $\beta$ & $B_{1}(\beta)$ & $B_{2}(\beta)$ \\
\hline$\overline{\mathrm{R} 1}$ & $\overline{\neg \dot{\neg} \phi}$ & $\bar{q}\{\phi\}$ & R4 & $\overline{\bar{\neg}\left(\phi_{1} \dot{\wedge} \phi_{2}\right)}$ & $\left\{\dot{\neg} \phi_{1}\right\}$ & 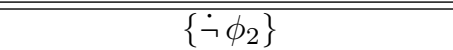 \\
\hline R2 & $\phi_{1} \dot{\wedge} \phi_{2}$ & $\left\{\phi_{1}, \phi_{2}\right\}$ & R5 & $\dot{\neg}\left(\phi_{1} \mathcal{U} \phi_{2}\right)$ & $\left\{\dot{\neg} \phi_{1}, \dot{\neg} \phi_{2}\right\}$ & $\left\{\phi_{1}, \dot{\neg} \phi_{2}, \dot{\neg} \bigcirc\left(\phi_{1} \mathcal{U} \phi_{2}\right)\right\}$ \\
\hline R3 & $\dot{\neg} \bigcirc \phi$ & $\{\bigcirc \neg \phi\}$ & R6 & $\phi_{1} \mathcal{U} \phi_{2}$ & $\left\{\phi_{2}\right\}$ & $\left\{\phi_{1}, \dot{\neg} \phi_{2}, \bigcirc\left(\left(\Gamma^{*} \dot{\wedge} \phi_{1}\right) \mathcal{U} \phi_{2}\right)\right\}$ \\
\hline
\end{tabular}

Intuitively, a tableau consists of a tree whose nodes are labeled with sets of formulas. The root is labeled with the set of formulas which has to be checked for satisfiability. Branches are built according to rules defined on the syntax of formulas (see Table 1 defining $\alpha$ and $\beta$ formulas). The basic idea is that a formula from a node is selected and, depending on its form, a rule of Table 1 is applied. $\beta$ formulas generate a bifurcation on the tree and there are specific rules for next and existential quantification formulas.

If all branches of the tree are closed (Definition 4.8), then the formula has no models. Otherwise, we can obtain a model from the open branches.

Definition 4.7 (csLTL tableau) A csLTL tableau for a finite set of formulas $\Phi$ is a tuple $\mathcal{T}_{\Phi}=\left(\right.$ Nodes, $\left.n_{\Phi}, L, B, R\right)$ such that:

1. Nodes is a finite non-empty set of nodes;

2. $n_{\Phi} \in$ Nodes is the initial node;

3. $L:$ Nodes $\rightarrow \wp(c s L T L)$ is the labeling function that associates to each node the formulas which are true in that node; the initial node is labeled with $\Phi$;

4. $B$ is the set of branches such that exactly one of the following points holds for every branch $b=n_{0}, \ldots, n_{i}, n_{i+1}, \ldots, n_{l} \in B$ and every $0 \leq i<l$ :

(a) for an $\alpha$-formula $\alpha \in L\left(n_{i}\right), L\left(n_{i+1}\right)=\{A(\alpha)\} \cup L\left(n_{i}\right) \backslash\{\alpha\}$;

(b) for a $\beta$-formula $\beta \in L\left(n_{i}\right), L\left(n_{i+1}\right)=\left\{B_{1}(\beta)\right\} \cup L\left(n_{i}\right) \backslash\{\beta\}$ and there exists another branch in $B$ of the form $b^{\prime}=n_{0}, \ldots, n_{i}, n_{i+1}^{\prime}, \ldots, n_{k}^{\prime}$ such that $L\left(n_{i+1}^{\prime}\right)=\left\{B_{2}(\beta)\right\} \cup L\left(n_{i}\right) \backslash\{\beta\}$;

(c) for an existential quantified formula $\dot{\exists}_{x} \phi^{\prime} \in L\left(n_{i}\right), L\left(n_{i+1}\right)=\left\{\phi^{\prime \prime}\right\} \cup L\left(n_{i}\right) \backslash$ $\left\{\dot{\exists}_{x} \phi^{\prime}\right\}$ where $\phi^{\prime \prime}:=\phi^{\prime}[y / x]$ with y fresh variable;

(d) in case $L\left(n_{i}\right)$ is a set formed only by elementary formulas, $L\left(n_{i+1}\right)=$ $\operatorname{next}\left(L\left(n_{i}\right)\right)$, where $\operatorname{next}(\Phi):=\{\phi \mid \bigcirc \phi \in \Phi\} \cup\{\dot{\neg} \phi \mid \dot{\neg} \bigcirc \phi \in \Phi\} \cup(\Phi \cap \mathbf{C})$.

Rules 4a and 4b are standard, replacing $\alpha$ and $\beta$-formulas with one or two formulas according to the matching pattern of rules in Table 1, except for Rule R6 that uses the so-called context $\Gamma^{*}$, which is defined in the following. The next operator used in Rule 4d is different from the corresponding one of PLTL since it also preserves the constraint formulas. This is needed for guaranteeing correctness in the particular setting of $t c c p$ where the store is monotonic. Finally, 
Rule 4c is specific for the $\dot{\exists}$ case: $\dot{\exists}_{x}$ is removed after renaming $x$ with a fresh variable $^{9}$.

Definition 4.8 A node in the tableau is inconsistent if it contains a couple of formulas $\phi, \dot{\neg} \phi$, or the formula false, or a constraint formula $\dot{\neg} c^{\prime}$ such that the merge $c$ of all the (positive) constraint formulas $c_{1}, \ldots, c_{n}$ in the node (i.e., $\left.c:=c_{1} \otimes \cdots \otimes c_{n}\right)$ is such that $c \vdash c^{\prime}$. A branch is closed if it contains an inconsistent node.

The last condition for inconsistence of a node is particular to the $c c p$ context.

We now describe the algorithm that automatically builds the csLTL tableau for a given set of formulas $\Phi$ (see [8] for the pseudocode). The construction consists in selecting at each step a branch that can be extended by using $\alpha$ or $\beta$ rules or $\dot{\exists}$ elimination. When none of these can be applied, the next operator is used to pass to the next stage. When dealing with eventualities, to determine the context $\Gamma^{*}$ in Rule R6, it is necessary to distinguish the eventuality that is being unfolded in the path. Given a node $n$ and $\phi \in L(n), \Gamma:=L(n) \backslash\{\phi\}$. Then, when Rule R6 is applied to a distinguished eventuality, we set $\Gamma^{*}:=\dot{V}_{\gamma \in \Gamma} \dot{\sim} \gamma$; otherwise $\Gamma^{*}:=$ true. The use of contexts is the mechanism to detect the loops that allows one to mark branches containing eventuality formulas as open or to generate inconsistent nodes and mark branches as closed. A node is marked as closed when it is inconsistent while is marked as open when (1) it is the last node of the branch and contains just constraint formulas or (2) the branch is cyclic and all the eventualities in the cycle have been already distinguished.

In order to ensure termination of the algorithm, it is necessary to use a fair strategy to distinguish eventualities, in the sense that every eventuality in an open branch must be distinguished at some point. This assumption and the fact that, given a finite set of initial formulas, there exists only a finite set of possible labels in a systematic tableau, imply termination of the tableau construction. Moreover, the constructed tableau is sound and complete. Therefore, to check the validity of a formula of the form $\psi \dot{\rightarrow} \phi$, with $\phi=\hat{\mathcal{S}}(p(\vec{x}))$ and $\psi=\hat{\mathcal{D}} \llbracket D \rrbracket_{\hat{\mathcal{S}}}(p(\vec{x}))$, we just have to build the tableau for its negation $\mathcal{T}_{\dot{\imath}(\psi \rightarrow \phi)}$ and check if it is closed or not. If it is, we have that $D$ is abstractly correct. Otherwise, we can extract from $\mathcal{T}_{\dot{\sim}(\psi \rightarrow \phi)}$ an explicit testimony $\varphi$ of the abstract incorrectness of $D$.

The construction of $\psi=\hat{\mathcal{D}} \llbracket D \rrbracket_{\hat{\mathcal{S}}}(p(\vec{x}))$ is linear in the size of $D$. The systematic tableau construction of $\dot{\neg}(\psi \dot{\rightarrow} \phi)$ (from what said in [16]) has worst case $O\left(2^{O\left(2^{|\dot{(\psi \rightarrow \phi}|)}\right)}\right)$. However, we believe that such bound for the worst-case asymptotic behavior is quite meaningless in this context, since it is not very realistic to think that the formulas of the specification should grow much (big formulas are difficult to comprehend and in real situations people would hardly try even to imagine them). Moreover, note that tableau explosion is due to nesting of eventualities and in practice really few eventualities are used in spec-

\footnotetext{
${ }^{9}$ The csLTL existential quantification does not correspond to the one of FO logic. It serves to model local variables, and $\dot{\exists}_{x} \phi$ can be seen just as $\phi$ where the information about $x$ is local.
} 
ifications. Therefore, in real situations, we do not expect that (extremely) big tableaux will be built.

\section{Related Work}

A Constraint Linear Temporal Logic is defined in [22] for the verification of a different timed concurrent language, called ntcc, which shares with tccp the concurrent constraint nature and the non-monotonic behavior. The restricted negation fragment of this logic, where negation is only allowed for state formulas, is shown to be decidable. However, no efficient decision procedure is given (apart from the proof itself). Moreover, the verification results are given for the locallyindependent fragment of $n t c c$, which avoids the non-monotonicity of the original language. In contrast, in this work, we address the problem of checking temporal properties for the full $t c c p$ language.

Some model-checking techniques have been defined for $t c c p$ in the past [14, $1,2,13]$. It is worth noting that the notions of correctness and completeness in these works are defined in terms of $\mathcal{F} \llbracket D \rrbracket$, i.e., in terms of the concrete semantics, and therefore their check requires a (potentially infinite) fixpoint computation. In contrast, the notions of abstractly incorrect declarations and

abstract uncovered elements are defined in terms of just one application of $\hat{\mathcal{D}} \llbracket D \rrbracket$ to $\hat{\mathcal{S}}$. Moreover, since $\hat{\mathcal{D}} \llbracket D \rrbracket$ is defined compositionally, all the checks are defined on each process declaration in isolation. Hence, our proposal can be used with partial sets of declarations. When a property is falsified, model checking provides a counterexample in terms of an erroneous execution trace, leaving to the user the problem of locating the source of the bug. On the contrary, we identify the faulty process declaration.

In [12], a first approach to the declarative debugging of a $c c p$ language is presented. However, it does not cover the particular extra difficulty of the nonmonotonicity behavior, common to all timed concurrent constraint languages. This makes our approach significantly different. Moreover, although they propose the use of LTL for the specification of properties, their formulation, based on the depth-k concretization function, complicates the task of having an efficient implementation.

Finally, this proposal clearly relates to the abstract diagnosis framework for tccp defined for Galois Insertions [5]. That work can compete with the precision of model checking, but its main drawback is the fact that the abstract domain did not allow to specify temporal properties in a compact way. In fact, specifications consisted of sets of abstract conditional traces. Thus, specifications were big and unnatural to be written. The use of temporal logic in this proposal certainly overcomes this problem. 


\section{Conclusion and Future Work}

We have defined an abstract semantics for tccp based on the domain of a linear temporal logic with constraints. The semantics is correct w.r.t. the behavior of the language.

By using this abstract semantics, we have defined a method to validate csLTL formulas for tccp sets of declarations. Since the abstract semantics cannot be defined by means of a Galois Connection, we cannot use the abstract diagnosis framework for tccp defined in [5], thus we devised (from scratch) a weak version of the abstract diagnosis framework based only on a concretization function $\gamma$. It works by applying $\hat{\mathcal{D}} \llbracket D \rrbracket$ to the abstract specification and then by checking the validity of the resulting implications (whether that computation implies the abstract specification). The computational cost depends essentially on the cost of that check of the implication.

We have also presented an automatic decision procedure for the csLTL logic, thus we can effectively check the validity of that implication. We are currently finishing to implement a proof of concept tool, which is available online at URL http://safe-tools.dsic.upv.es/tadi/, that realizes the proposed instance. Then we would be able to compare with other tools and assess the "real life" goodness of our proposal.

In the future, we also plan to explore other instances of the method based on logics for which decision procedures or (semi)automatic tools exists. This proposal can also be immediately adapted to other concurrent (non-monotonic) languages (like $t c c$ and $n t c c$ ) once a suitable fully abstract semantics has been developed.

\section{References}

[1] M. Alpuente, M. Falaschi, and A. Villanueva. A Symbolic Model Checker for tccp Programs. In First International Workshop on Rapid Integration of Software Engineering Techniques (RISE 2004), Revised Selected Papers, volume 3475 of Lecture Notes in Computer Science, pages 45-56. SpringerVerlag, 2005.

[2] M. Alpuente, M.M. Gallardo, E. Pimentel, and A. Villanueva. A Semantic Framework for the Abstract Model Checking of tccp Programs. Theoretical Computer Science, 346(1):58-95, 2005.

[3] M. Alpuente, M.M. Gallardo, E. Pimentel, and A. Villanueva. Verifying Real-Time Properties of tccp Programs. Journal of Universal Computer Science, 12(11):1551-1573, 2006.

[4] E. M. Clarke and E. A. Emerson. Design and synthesis of synchronization skeletons using branching-time temporal logic. In Logic of Programs, Workshop, pages 52-71, London, UK, 1982. Springer-Verlag. 
[5] M. Comini, L. Titolo, and A. Villanueva. Abstract Diagnosis for Timed Concurrent Constraint programs. Theory and Practice of Logic Programming, 11(4-5):487-502, 2011.

[6] M. Comini, L. Titolo, and A. Villanueva. A Condensed Goal-Independent Bottom-Up Fixpoint Modeling the Behavior of tccp. Technical report, DSIC, Universitat Politècnica de València. Available at http://riunet. upv.es/handle/10251/34328, 2013.

[7] M. Comini, L. Titolo, and A. Villanueva. Towards an Effective Decision Procedure for LTL formulas with Constraints. In 23rd Workshop on Logic-based methods in Programming Environments (WLPE 2013), volume abs/1308.2055, Istanbul, 2013. arXiv.org.

[8] M. Comini, L. Titolo, and A. Villanueva. Abstract Diagnosis for tccp using a Linear Temporal Logic. Technical report, Universitat Politècnica de València. Available at http://riunet.upv.es/handle/10251/8351, 2014.

[9] F. S. de Boer, M. Gabbrielli, and M. C. Meo. A Timed Concurrent Constraint Language. Information and Computation, 161(1):45-83, 2000.

[10] F. S. de Boer, M. Gabbrielli, and M. C. Meo. A Temporal Logic for Reasoning about Timed Concurrent Constraint Programs. In TIME '01: Proceedings of the Eighth International Symposium on Temporal Representation and Reasoning (TIME'01), page 227, Washington, DC, USA, 2001. IEEE Computer Society.

[11] F. S. de Boer, M. Gabbrielli, and M. C. Meo. Proving correctness of Timed Concurrent Constraint Programs. CoRR, cs.LO/0208042, 2002.

[12] M. Falaschi, C. Olarte, C. Palamidessi, and F. D. Valencia. Declarative Diagnosis of Temporal Concurrent Constraint Programs. In V. Dahl and I. Niemelä, editors, Logic Programming, 23rd International Conference, ICLP 2007, Proceedings, volume 4670 of Lecture Notes in Computer Science, pages 271-285. Springer-Verlag, 2007.

[13] M. Falaschi, A. Policriti, and A. Villanueva. Modeling concurrent systems specified in a temporal concurrent constraint language-I. Electronic Notes in Theoretical Computer Science, 48:197-210, 2001.

[14] M. Falaschi and A. Villanueva. Automatic verification of timed concurrent constraint programs. Theory and Practice of Logic Programming, 6(3):265$300,2006$.

[15] J. Gaintzarain, M. Hermo, P. Lucio, and M. Navarro. Systematic semantic tableaux for PLTL. Electronic Notes in Theoretical Computer Science, 206:59-73, 2008. 
[16] J. Gaintzarain, M. Hermo, P. Lucio, M. Navarro, and F. Orejas. Dual Systems of Tableaux and Sequents for PLTL. The Journal of Logic and Algebraic Programming, 78(8):701-722, 2009.

[17] Z. Manna and A. Pnueli. The temporal logic of reactive and concurrent systems - specification. Springer, 1992.

[18] C. Palamidessi and F. D. Valencia. A Temporal Concurrent Constraint Programming Calculus. In 7th International Conference on Principles and Practice of Constraint Programming (CP'01), volume 2239 of Lecture Notes in Computer Science, pages 302-316. Springer, 2001.

[19] J. P. Queille and J. Sifakis. Specification and verification of concurrent systems in CESAR. In M. Dezani-Ciancaglini and U. Montanari, editors, Symposium on Programming, volume 137 of Lecture Notes in Computer Science, pages 337-351. Springer, 1982.

[20] V. A. Saraswat. Concurrent Constraint Programming Languages. PhD thesis, Carnegie-Mellon University, January 1989.

[21] V. A. Saraswat. Concurrent Constraint Programming. The MIT Press, Cambridge, Mass., 1993.

[22] F. D. Valencia. Decidability of infinite-state timed CCP processes and first-order LTL. Theoretical Computer Science, 330(3):577-607, 2005. 